\title{
DETERIORATION OF THE MOLECULAR FIELD ${ }^{\ddagger}$
}

\author{
H. FALK* and TH.W. RUIJGROK \\ Instituut voor Theoretische Fysica, Rijksuniversiteit te Utrecht, \\ Utrecht, The Netherlands
}

Received 9 July 1974

\section{Synopsis}

The molecular-field model of an Ising ferromagnet is modified by cutting interaction bonds so as to leave a system of $M$ chains with long-range interactions between nearest-neighbor chains only. Equilibrium properties of the model, such as the critical temperature

$$
k_{\mathrm{B}} T_{\mathrm{c}} / J=[2 \cos \{\pi /(M+1)\}] / M \quad(M \geq 2),
$$

are established analytically, and numerical results are also given for $M=6$.

1. Introduction. In this work we begin with $N$ Ising spins and a hamiltonian $H$ which, in the exact thermodynamic limit, gives the classical, molecular-field behavior of an Ising ferromagnet. We modify the hamiltonian by selectively "cutting" interaction bonds so that what remains is a hamiltonian $H_{M}$ describing $M$ chains of $n$ spins per chain $(N=M n)$ such that the long-range interactions are between nearest-neighbor chains only. After taking the double limit $N \rightarrow \infty$, $n \rightarrow \infty$ with $M$ finite, we obtain a system of equations of state for the magnetizations $m_{r}(r=1,2, \ldots, M)$ of the chains. Our purpose here is to present exact properties of (stable and unstable) solutions of the system. The results relate to understanding the equilibrium behavior and also contribute to an investigation of the dynamics of the approach to equilibrium.

The next section contains a description of the model, the resulting system of equations of state, and a basis for the dynamical investigation in terms of a freeenergy functional.

In section 3 general equilibrium properties are established and, for convenience, are separated into the mathematical format of "theorems and proofs". This should

\# Summary presented at the Tenth Symposium on Many-Body Theory and Statistical Mechanics, Bedford College, London, 17 May 1974.

\# Permanent address: Department of Physics, City College of the CUNY, New York, N.Y. 10031, USA. 
assist the reader in focusing on the main points and in deciding what is actually proved. In that section we obtain the ferromagnetic Curie temperature

$$
k_{\mathrm{B}} T_{\mathrm{c}} / J=[2 \cos \{\pi /(M+1)\}] / M \quad(M \geq 2),
$$

which, as expected, decreases monotonically as the number of chains increases; i.e., as the number of cut bonds increases. It is proved that the trivial solution is the only solution and is stable for $T>T_{\mathrm{c}}$, but is a hypersaddle point for $T \leq T_{\mathrm{c}}$. Conncctions are also made betwecn stability and the Griffiths inequalities, as well as between the loss of stability and the classical $1 /\left|T-T_{\mathrm{c}}\right|$ divergence of the susceptibility.

In section 4 properties of symmetric solutions $\left(m_{r}=m_{M-r+1}\right)$ are presented for $M$ an even integer. For $T \rightarrow T_{\mathfrak{c}}^{-}$the explicit value of $m_{r} / m_{1}$ for $r=1,2, \ldots, M / 2$ is given in terms of the eigenvector associated with the maximum eigenvalue of a matrix. It is proved for $0<T<T_{\mathrm{c}}$ that the (positive) ferromagnetic solution satisfies the ordering: $0<m_{1}<m_{2}<\cdots<m_{M / 2}$ and a functional lower bound is developed for $m_{M / 2}(T)$.

In section 5 attention is restricted to six chains $(M=6)$ for which additional properties of symmetric solutions are obtained. In particular, the stability of the pair of ferromagnetic solutions is proved. The equations are solved numerically and magnetization curves $m_{1}(T) \equiv m_{6}(T), m_{2}(T) \equiv m_{5}(T), m_{3}(T) \equiv m_{4}(T)$ are given and discussed.

2. The model. Consider a set of $N$ spins $\sigma_{i}= \pm 1, i=1,2, \ldots, N$, then for $J>0$ the hamiltonian associated with the molecular-field theory of an Ising ferromagnet $^{1}$ ) is

$$
H=-(J / N) \sum_{1 \leqslant i<j \leqslant N} \sigma_{i} \sigma_{j}
$$

Partition the set of $N$ spins into $M$ subsets of spins $\sigma_{r s}$ where $r=1,2, \ldots, M$; $s=1,2, \ldots, n$ and

$$
n=N / M
$$

is a positive integer. Geometrically, the spins may be considered as arranged in $M$ rows with $n$ spins in each row.

Now cut all interaction bonds between spins in the same row; i.e., cut all bonds between spins with the same $r$. Also cut all interaction bonds between spins in rows which are not nearest-neighbour rows. The hamiltonian for the resulting system is given by

$$
H_{M}=-(J / M) n \sum_{1 \leqslant r \leqslant M-1} \xi_{r} \xi_{r+1},
$$


where

$$
\xi_{r}=(1 / n) \sum_{1 \leqslant s \leqslant n} \sigma_{r s}
$$

With $\sigma$ denoting the set of $2^{N}$ spin configurations, the canonical ensemble average of $\xi_{r}$ is written

$$
m_{r}^{(N)}=\sum_{\sigma}\left[\xi_{r} \exp \left(-\beta H_{M}\right)\right] / \sum_{\sigma} \exp \left(-\beta H_{M}\right)
$$

Implicit in the definition is a term which breaks spin-reversal symmetry and is taken to $0^{+}$after the following limit.

In the limit $N \rightarrow \infty, n \rightarrow \infty$ with $M=N / n=\mathcal{O}(1)$ the standard asymptotic methods $^{1}$ ) provide the system of equations

$$
m_{r}=\tanh \left[\alpha\left(m_{r-1}+m_{r+1}\right)\right] \quad(r=1,2, \ldots, M),
$$

where

$$
m_{0} \equiv m_{M+1} \equiv 0
$$

and $m_{r}$ denotes the value of $m_{r}^{(N)}$ in the above limit.

The parameter $\alpha$ is given by

$$
\alpha=\beta J / M_{1}>0
$$

where in terms of the Boltzmann constant $k_{\mathrm{B}}$ and the absolute temperature $T$, $k_{\mathrm{B}} T=1 / \beta$.

The equation of state given above has multiple solutions. Each solution will be an extremum of the free-energy functional

$$
\begin{aligned}
\varphi= & \frac{1}{M} \sum_{1 \leqslant r \leqslant M}\left[\left(\frac{1+m_{r}}{2}\right) \ln \left(\frac{1+m_{r}}{2}\right)+\left(\frac{1-m_{r}}{2}\right) \ln \left(\frac{1-m_{r}}{2}\right)\right. \\
& \left.-\alpha m_{r} m_{r+1}\right],
\end{aligned}
$$

since

$$
\tanh \left[\alpha\left(m_{r-1}+m_{r+1}\right)\right]-m_{r}=-M g_{r} \partial \varphi / \partial m_{r}
$$

where

$$
g_{r}=\frac{\tanh \left[\alpha\left(m_{r-1}+m_{r+1}\right)\right]-m_{r}}{\alpha\left(m_{r-1}+m_{r+1}\right)-\tanh ^{-1} m_{r}}>0 .
$$


The determination of the equilibrium thermodynamic state can be carried out by introducing a Zeeman term $-\sum_{r} h m_{r}$ into $\varphi$ and requiring that $h \rightarrow 0^{+}$. Alternatively, one could follow the approach of Lebowitz and Penrose $\left.{ }^{1}\right)$. Since, for the system described by $H$, these methods provide one with the same ferromagnetic state for which Griffiths'2) inequalities obtain, we anticipate a similar equivalence and therefore investigate the possible solutions of eq. (2.6) and identify the ferromagnetic solution.

The latter approach has an interesting advantage when one studies the model from a dynamical view point by writing ${ }^{* 3}$ ),

$$
\begin{aligned}
\mathrm{d} m_{r} / \mathrm{d} t & =-M g_{r} \partial \varphi / \partial m_{r} \\
& =\tanh \left[\alpha\left(m_{r-1}+m_{r+1}\right)\right]-m_{r}
\end{aligned}
$$

and noticing that

$$
\mathrm{d} \varphi / \mathrm{d} t \leq 0,
$$

with equality when and only when an equilibrium of the dynamical system has been reached. But $\mathrm{d} m_{r} / \mathrm{d} t=0$ when and only when $\partial \varphi / \partial m_{r}=0$. Thus, the freeenergy functional $\varphi$ is essentially a Lyapunov function which contains the entire stability structure of the above dynamical system. Given all solutions of the equation of state, eq. (2.6), one can obtain an overall picture of the approach to thermodynamic equilibrium. In the present work attention will be restricted to the problem of solving and studying the stability of the solutions of eq. (2.6). Dynamical results will be presented in another paper $^{4}$ ).

3. Equilibrium properties (general). A convenient parameter which serves to characterize eq. (2.6) is

$$
\tau \equiv 1 / M \alpha=k_{\mathrm{B}} T / J
$$

with which we associate a critical value

$$
\tau_{\mathbf{c}} \equiv[2 \cos \{\pi /(M+1)\}] / M .
$$

We now establish an important property of eq. (2.6).

Theorem 3.1: For $\tau \geq \tau_{\mathrm{c}}$ the only solution of eq. (2.6) for which $m_{r} \geq 0$ is the trivial solution $\left(m_{r}=0\right.$ for $\left.r=1,2, \ldots, M\right)$.

* This phenomenological idea of relating the approach to equilibrium to the negative freecnergy gradient can beput into a more microscopic context by using a master equation given in ref. 3 and by appropriately selecting the transition probabilities. 
Remark: We will subsequently exhibit a nontrivial, stable, ferromagnetic solution $m_{r}>0$ for $0<\tau<\tau_{\mathrm{c}}$; consequently, $T_{\mathrm{c}}=J \tau_{\mathrm{c}} / k_{\mathrm{B}}$ is the ferromagnetic Curie (critical) temperature for the spin system described by the hamiltonian $H_{M}$.

Notice that as $M$ increases from the value $2, T_{\mathrm{c}}$ decreases, as expected, since more bonds have been cut. Ultimately, the molecular field deteriorates entirely and one is left with a nearest-neighbour Ising chain for which $T_{\mathrm{c}}=0$.

Proof: Introduce the $M \times M$ matrix

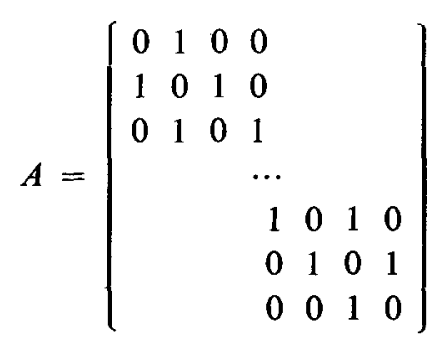

and the row vectors

$$
\begin{aligned}
& \langle m|=\left(m_{1}, m_{2}, \ldots, m_{M}\right), \\
& \langle\varepsilon|=\left(\varepsilon_{1}, \varepsilon_{2}, \ldots, \varepsilon_{M}\right),
\end{aligned}
$$

where

$$
\varepsilon_{r}=\tanh \left[\alpha\left(m_{r-1}+m_{r+1}\right)\right]-\alpha\left(m_{r-1}+m_{r+1}\right) .
$$

Since $\alpha>0$, it follows from the convexity of the tanh that $\varepsilon_{r} \leq 0$ with equality if and only if $m_{r-1}+m_{r+1}=0$.

Denote the column vectors corresponding to the transpose of $\langle m|$ and $\langle\varepsilon|$ by $|m\rangle$ and $|\varepsilon\rangle$, respectively; then eq. (2.6) is equivalent to

$$
(I-\alpha A)|m\rangle=|\varepsilon\rangle .
$$

Now the eigenvalues of $A \operatorname{are}^{5}$ )

$$
\lambda_{k}=2 \cos \{k \pi /(M+1)\}, \quad k=1,2, \ldots, M,
$$

therefore, $I-\alpha A$ is positive-definite if and only if

$$
1-\alpha \lambda_{1}>0 \text {. }
$$

But $\alpha=\beta J / M$; so that the inequality is equivalent to

$$
\tau>\tau_{\mathrm{c}} \quad \text { with } \quad \alpha_{\mathrm{c}}=1 / \lambda_{1} .
$$


On the other hand, the off-diagonal elements of $l-\alpha A$ are $\leq 0$. Thus, for $\tau>\tau_{\mathrm{c}}, I-\alpha A$ is a positive-definite matrix with nonpositive off-diagonal elements. A theorem of Stieltjes-Ostrowski (see ref. 6 for a discussion of this theorem) states that the inverse $(I-\alpha A)^{-1}$ has all elements $\geq 0$. For $\tau>\tau_{\mathrm{c}}$ we are therefore confronted with the situation

$$
|m\rangle=(I-\alpha A)^{-1}|\varepsilon\rangle,
$$

where the right-hand side of the equation contains a vector $(I-\alpha A)^{-1}|\varepsilon\rangle$ with nonpositive elements. This proves the theorem.

Remark: Since eq. (2.6) has the symmetry property that given any solution $|\hat{m}\rangle$, $-|\hat{m}\rangle$ is also a solution for the same $\alpha$, the above statement of the theorem is also valid if one replaces $m_{r} \geq 0$ by $m_{r} \leq 0$.

Definition: The stability of any solution $|\hat{m}\rangle$ of eq. (2.6) is established by showing that the matrix $D$ defined by

$$
D_{r s}=\alpha^{-1} M \partial^{2} \varphi / \partial m_{r} \partial m_{s} \quad(|m\rangle=|\hat{m}\rangle),
$$

is positive-definite. If $D$ is not positive-definite, then the solution $|\hat{m}\rangle$ is not stable.

Theorem 3.2: For $\tau>\tau_{\mathrm{c}}$ the trivial solution of eq. (2.6) is stable, and for $\tau \leq \tau_{\mathrm{c}}$ it is not stable.

Proof: Differentiation of $\varphi$ gives the matrix elements of $D$ :

$$
\alpha^{-1} M \frac{\partial^{2} \varphi}{\partial m_{r} \partial m_{s}}=\left[\begin{array}{cc}
{\left[\alpha\left(1-m_{r}^{2}\right)\right]^{-1}} & \text { for } \quad r=s, \\
-1 & \text { for } \quad r=s \pm 1, \\
0 & \text { otherwise. }
\end{array}\right.
$$

For the trivial solution, $|\hat{m}\rangle=0, D$ has the particularly simple form

$$
\alpha D=I-\alpha A \quad(|\hat{m}\rangle=0) .
$$

Referring to the proof of the previous theorem, one sees that $D$ is positive-definite if and only if $\tau>\tau_{\mathrm{c}}$. The trivial solution is therefore stable if and only if $\tau>\tau_{\mathrm{c}}$.

Theorem 3.3: For $\tau>\tau_{\mathrm{c}}$ the only solution of eq. (2.6) in the domain $0 \leq m_{r}<1$ is the trivial solution, and it gives the absolute minimum of $\varphi$.

Proof: With $D_{0}$ denoting $D$ at $|m\rangle=0$, Taylor expansion of $\varphi$ for $0 \leq m_{r}<1$ gives

$$
\varphi(|m\rangle)=\varphi(0)+\left[\alpha\left\langle m\left|D_{0}\right| m\right\rangle+2 \sum_{1 \leqslant r \leqslant M} \sum_{2 \leqslant l \leqslant \infty} m_{r}^{2 l} /(2 l)(2 l-1)\right] / 2 M .
$$


But, for $\tau>\tau_{\mathrm{c}}, D_{0}$ is positive-definite so that $\left\langle m\left|D_{0}\right| m\right\rangle$ and the double sum is $\geq 0$ (equality if and only if $|m\rangle=0$ ). Thus, for $\tau>\tau_{\mathrm{c}}, \varphi(|m\rangle)>\varphi(0)$ for all $|m\rangle \neq 0$, which shows that the trivial solution yields the absolute minimum of the free-energy functional above the critical temperature.

Furthermore, for $\tau>\tau_{\mathrm{c}}$

$$
\sum_{1 \leqslant r \leqslant M} m_{r} \partial \varphi / \partial m_{r} \geq 0
$$

with equality if and only if $|m\rangle=0 . \varphi$ is therefore strictly increasing on successively larger spheres centred at the origin $(|m\rangle=0)$; consequently, for $\tau>\tau_{\mathfrak{c}}$, $\varphi$ has no extrema other than at the origin. This establishes that above the critical temperature the only solution of eq. (2.6) in the given domain is the trivial solution (and generalizes theorem 3.1).

Remark: The stability of a solution $|\hat{m}\rangle$ of course implies that at $|m\rangle=|\hat{m}\rangle$ the function $\varphi$ achieves a minimum in the $M$-dimensional space with axes $m_{1}, m_{2}$, $\ldots, m_{M}$. One might guess that since the trivial solution is not stable for $\tau \leq \tau_{\mathbf{c}}$ the function $\varphi$ is a maximum at $|m\rangle=0$ for $\tau \leq \tau_{\mathrm{c}}$. That would be incorrect, however, because some of the eigenvalues of $A$ are negative and the eigenvalues of $I-\alpha A$ can therefore not all be negative. Geometrically, in the $M+1$-dimensional space with axes $m_{1}, m_{2}, \ldots, m_{M}, \varphi$, the point $(0,0, \ldots, 0, \varphi(0))$ is a hypersaddle point for $\tau \leq \tau_{c}$ and a minimum for $\tau>\tau_{\mathrm{c}}$.

Theorem 3.4: Any solution $|m(\alpha)\rangle$ of eq. (2.6) satisfies

$$
\alpha D \mathrm{~d}|m(\alpha)\rangle / \mathrm{d} \alpha=A|m(\alpha)\rangle \text {. }
$$

Proof: Differentiate eq. (2.6) and use eq. (3.13).

Remark: For the ferromagnetic solution, $|m(\alpha)\rangle$ is a vector with all elements positive. When that solution is stable, $D$ is positive-definite; therefore, $D^{-1}$ has all positive elements ${ }^{6}$ ) and $\mathrm{d}|m(\alpha)\rangle / \mathrm{d} \alpha$ is a positive vector in accord with Griffiths' second inequality ${ }^{2}$ ).

Definition: If the term $n \sum_{1 \leqq r \leqq M} h_{r} \xi_{r}$ is inserted into the equation for $-\beta H_{M}$ [sce eq. (2.3)], then eq. (2.6) is modified:

$$
m_{r}=\tanh \left[\alpha\left(m_{r-1}+m_{r+1}\right)+h_{r}\right]
$$

The susceptibility matrix $\chi$ with elements $\chi_{r s}$ is defined by

$$
\chi_{r s}=\partial m_{r} / \partial h_{s}
$$


Theorem 3.5: Any solution of eq. (2.6) satisfies

$$
\alpha D_{\chi}=I
$$

Proof: Differentiate eq. (2.6) and use eq. (3.13).

Remark: Again the stable, ferromagnetic solution satisfies Griffiths' inequalities ${ }^{2}$ ); in this case by ensuring that each element of the susceptibility matrix is positive.

That the phase transition is of the "classical" type can be seen as follows. Look first at eq. (3.14) for the stability matrix when $\tau>\tau_{\mathrm{c}}\left(\alpha<\alpha_{\mathrm{c}}\right)$. As $\tau \rightarrow \tau_{\mathrm{c}}^{+}$the smallest eigenvalue of $D$ becomes zero. But that eigenvalue is $1 / \alpha-\lambda_{1}=1 / \alpha-1 / \alpha_{c}$. From theorem 3.5 it is clear that the vanishing of that eigenvalue causes the elements of the susceptibility matrix to diverge as $1 /\left(\tau-\tau_{\mathrm{c}}\right)$.

The same analysis applies for $\tau \rightarrow \tau_{\mathrm{c}}^{-}$and leads to the standard classical singularities for the magnetization and susceptibility.

4. Equilibrium properties (symmetric). For convenience we now restrict the study to an even number ( $M$ an even positive integer) of chains, and investigate ferromagnetic $\left(m_{r} \geq 0\right)$ solutions which have the symmetry

$$
m_{r}=m_{M-r+1} \quad(r=1,2, \ldots, M) .
$$

The system of equations, eq. (2.6), becomes

$$
\begin{aligned}
& m_{r}=\left[\begin{array}{ll}
\tanh \left[\alpha\left(m_{r-1}+m_{r+1}\right)\right] & (r=1,2, \ldots, M / 2), \\
m_{M-r+1} & (r=M / 2+1, \ldots, M),
\end{array}\right. \\
& m_{\mathrm{o}}=0 \text {. }
\end{aligned}
$$

Notice that $m_{M / 2+1}=m_{M / 2}$; thus, for $r=1,2, \ldots, M / 2$ one has a closed system of equations, and the free-energy functional $\psi$ corresponding to $\varphi$ is

$$
\begin{aligned}
\psi= & \frac{2}{M}\left\{\sum_{1 \leqslant r \leqslant M / 2}\left[\left(\frac{1+m_{r}}{2}\right) \ln \left(\frac{1+m_{r}}{2}\right)+\left(\frac{1-m_{r}}{2}\right) \ln \left(\frac{1-m_{r}}{2}\right)\right]\right. \\
& \left.-\alpha\left(m_{1} m_{2}+m_{2} m_{3}+\cdots+m_{M / 2-1} m_{M / 2}+\frac{1}{2} m_{M / 2}^{2}\right)\right\}
\end{aligned}
$$

Theorem 4.1: For $\tau \rightarrow \tau_{\mathrm{c}}^{-}$the ferromagnetic solution of eq. (4.1) satisfies the equations

$$
\begin{aligned}
m_{n} / m_{n+s} & =\sin \{n \pi /(M+1)\} / \sin \{(n+s) \pi /(M+1)\} \\
& =[\cos \{s \pi /(M+1)\}+\sin \{s \pi /(M+1)\} \cot \{n \pi /(M+1)\}]^{-1}
\end{aligned}
$$

for $1 \leq n+s \leq M / 2,1 \leq n \leq M / 2$. 
Remark: The above equations show the relative magnetization of the end chains and the central chains for $\tau$ just below $\tau_{\mathbf{c}}$. For large $M$ one has the striking result

$$
m_{M / 2} / m_{1} \sim(M+1) / \pi .
$$

Proof: Introduce the $M / 2 \times M / 2$ matrices

$$
A(\delta)=\left[\begin{array}{rrrrrrrr}
0 & 1 & 0 & 0 & & & \\
1 & 0 & 1 & 0 & & & \\
0 & 1 & 0 & 1 & & & \\
& & & \cdots & & & \\
& & & & 1 & 0 & 1 & 0 \\
& & & 0 & 1 & 0 & 1 \\
& & & & 0 & 0 & 1 & \delta
\end{array}\right] \quad \text { for } \quad \delta= \pm 1
$$

The eigenvalues $\lambda_{k}(\delta)$ are given $b^{5}$ )

$$
\begin{aligned}
& \lambda_{k}(1)=2 \cos \{(2 k-1) \pi /(M+1)\} \quad(k=1,2, \ldots, M / 2), \\
& \lambda_{k}(-1)=2 \cos \{2 k \pi /(M+1)\} \quad(k=1,2, \ldots, M / 2) .
\end{aligned}
$$

Notice that the spectra of $A(-1)$ and $A(1)$ interlace and that the union of their spectra is the spectrum of $A$ [see eq. (3.8)]. Also notice that the maximum eigenvalue of $A$ is contained in the spectrum of $A(1)$. One can, in fact, show ${ }^{*}$ that $A$ is equivalent to the block-diagonal matrix, diag $(A(1), A(-1))$.

The eigenvectors $\left|\lambda_{k}(1)\right\rangle$ are readily found by solving the difference equation

$$
\lambda_{k}(1) u_{n, k}=u_{n+1, k}+u_{n-1, k} \quad(k, n=1,2, \ldots, M / 2),
$$

with the boundary conditions

$$
\begin{aligned}
& u_{0 k}=0, \\
& u_{M / 2+1, k}=u_{M / 2, k} .
\end{aligned}
$$

The solution

$$
u_{n, k}=a_{k}(-1)^{n(k+1)} \sin \{n k \pi /(M+1)\},
$$

is the $n$th element of the $k$ th eigenvector $\left|\lambda_{k}(1)\right\rangle$ where $a_{k}$ is a normalizing factor which we take to be positive.

Now the eigenvector associated with the maximum eigenvalue $\lambda_{1}(1)$ has all positive elements. Since it must be orthogonal to all other eigenvectors of $A(1)$, it is therefore the only eigenvector with all positive elements.

* We are indebted to Dr. J.Groeneveld for this helpful point. 
With those facts in mind consider eq. (4.1) for $\tau$ just less than $\tau_{\mathrm{c}}$ (recall: $\left.\tau_{\mathrm{c}}=1 / M \alpha_{\mathrm{c}}\right)$ :

$$
|m\rangle=\alpha_{\mathrm{c}} A(1)|m\rangle+\cdots,
$$

where the suppressed terms are of $\mathscr{O}\left(m_{1}^{3}\right)$ and will play no role in the limit $\tau \rightarrow \tau_{\mathrm{c}}^{-}$. But we also have

$$
\lambda_{1}(1)\left|\lambda_{1}(1)\right\rangle=A(1)\left|\lambda_{1}(1)\right\rangle,
$$

where

$$
\lambda_{1}(1)=1 / \alpha_{c}
$$

Clearly then, as $\tau \rightarrow \tau_{\mathrm{c}}^{-},|m\rangle$ approaches a vector proportional to the eigenvector $\left|\lambda_{1}(1)\right\rangle$ with elements $u_{n, 1}=a_{1} \sin \{n \pi /(M+1)\}$. The ratios given in the theorem follow immediately.

Theorem 4.2: The ferromagnetic (nontrivial) solution of eq. (4.1) for $\tau<\tau_{\mathrm{c}}$ satisfies the ordering relation

$$
0<m_{1}<m_{2}<m_{3}<\cdots<m_{M / 2} \text {. }
$$

Remark: From a physical point of view one would expect the thermodynamic, ferromagnetic solution to exhibit the above ordering, since the end chains each have only one nearest neighbor and should have a smaller average magnetization than the successive internal chains. The magnetization should "build up" monotonically from the outer chains toward the center.

Proof: Written in detail eq. (4.1) gives

$$
\begin{aligned}
& m_{1}=\tanh \alpha m_{2}, \\
& m_{2}=\tanh \alpha\left(m_{1}+m_{3}\right), \\
& m_{3}=\tanh \alpha\left(m_{2}+m_{4}\right), \\
& m_{4}=\tanh \alpha\left(m_{3}+m_{5}\right) \\
& m_{5}=\tanh \alpha\left(m_{4}+m_{6}\right), \\
& \vdots \\
& m_{M / 2}-1=\tanh \alpha\left(m_{M / 2-2}+m_{M / 2}\right), \\
& m_{M / 2}=\tanh \alpha\left(m_{M / 2-1}+m_{M / 2}\right) .
\end{aligned}
$$

We are studying ferromagnetic solutions $\left(m_{r}>0\right)$. Inspection of the equations shows that if any $m_{j}=0$, then all $m_{r}=0$. We have shown that as $\tau \rightarrow \tau_{\mathrm{c}}^{-}$the 
ferromagnetic solution has the ordering given in the theorem. The only way that the ordering may change as $\alpha$ increases continuously is for two nearest-neighboring $m$ 's to become equal. But if that happens, it is easy to see that all $m$ 's become zero; i.e., one has the trivial solution. This establishes for the ferromagnetic, nontrivial solution that the above ordering is preserved for $\tau<\tau_{\mathrm{c}}$.

Theorem 4.3: The ferromagnetic solution of eq. (4.1) satisfies the inequality $m_{M / 2} \geq m^{*}$ where $m^{*}$ is the largest solution of the equation

$$
m=\tanh \left(\alpha m / \alpha_{c}\right) .
$$

Proof: Define the row vector

$$
\left\langle\tanh ^{-1}(m)\right|=\left(\tanh ^{-1}\left(m_{1}\right), \ldots, \tanh ^{-1}\left(m_{M / 2}\right)\right)
$$

then eq. (4.1) can be written in terms of column vectors:

$$
\alpha A(1)|m\rangle=\left|\tanh ^{-1}(m)\right\rangle \text {. }
$$

Take the inner product with the maximum eigenvector $\left|\lambda_{1}(1)\right\rangle$ and use eqs. (4.6), (3.8), (3.10) and (4.11):

$$
\left(\alpha / \alpha_{c}\right)\left\langle\lambda_{1}(1) \mid m\right\rangle=\left\langle\lambda_{1}(1) \mid \tanh ^{-1}(m)\right\rangle,
$$

or, equivalently,

$$
\sum_{1 \leqslant r \leqslant M / 2}\left[\left(\alpha m_{r} / \alpha_{c}\right)-\tanh ^{-1}\left(m_{r}\right)\right] \sin \{r \pi /(M+1)\}=0 .
$$

Consider the function

$$
f(z)=\eta z-\tanh ^{-1}(z) \quad\left(0 \leq z<1 ; 1 \leq \alpha / \alpha_{\mathrm{c}} \equiv \eta\right)
$$

Then $f(z)$ has roots at 0 and $m^{*}$ only and $f^{\prime \prime}(z) \leq 0$ for $z \geq 0$. This means that $f(z)>0$ for $0<z<m^{*}$ and $f(z)<0$ for $m^{*}<z<1$. Now assume that $f\left(m_{M / 2}\right)>0$, then since $0<m_{1}<m_{2}<\cdots<m_{M / 2}$ and $\sin \{r \pi /(M+1)\}>0$ for $r=1,2, \ldots, M / 2$, eq. (4.21) would have no solution. However, we know that a nontrivial ferromagnetic solution exists for $\alpha>\alpha_{\mathrm{c}}$; therefore, we must have $f\left(m_{M / 2}\right) \leq 0$ and that is the content of the theorem.

Theorem 4.4: The ferromagnetic solution of eq. (4.1) satisfies the inequality $m_{1} \leq m^{*}$ where $m^{*}$ is defined above.

Proof: As for the preceding theorem but assume $f\left(m_{1}\right)<0$ and arrive at a contradiction. 
5. Equilibrium properties ( 6 chains). The remaining discussion will involve solutions of eq. (4.1) (the symmetric system) for $M=6$. Analytic and numerical results which supplement and confirm those in previous sections will be given.

The equations are

$$
\begin{aligned}
& m_{1}=\tanh \alpha m_{2}, \\
& m_{2}=\tanh \alpha\left(m_{1}+m_{3}\right), \\
& m_{3}=\tanh \alpha\left(m_{2}+m_{3}\right), \\
& m_{1}=m_{6}, \quad m_{2}=m_{5}, \quad m_{3}=m_{4} .
\end{aligned}
$$

We first give an alternative proof of theorem 4.2 ; i.e., we show that $0<m_{1}<m_{2}<m_{3}$ for the nontrivial ferromagnetic solution $\left(m_{r}>0\right)$ : By inspection, $m_{3}>m_{1}$. Now assume $m_{2}<m_{1}$, then the last equation implies: $m_{3}<\tanh \left(m_{1}+m_{3}\right)=m_{2}$ which is $<m_{1}$ by hypothesis. But $m_{3}<m_{1}$ contradicts the fact that $m_{3}>m_{1}$; consequently, $m_{2}>m_{1}$ and, with the last equation, one concludes that $m_{3}>m_{2}$. The complete order $0<m_{1}<m_{2}<m_{3}$ thus follows for the nontrivial ferromagnetic solution.

Theorem 5.1: For the nontrivial, ferromagnetic solution of eq. $(5.1)^{\ddagger}$

$$
0<m_{3}-m_{2}<m_{2}-m_{1}<m_{1} m_{3} /\left(m_{1}+m_{2}\right) \text {. }
$$

Remark: This is another physically intuitive result which states that the change of magnetization with distance (chain number) decreases as one goes from the outside toward the central chains, as expected for a "surface effect".

Proof: We eliminate $\alpha$ from eq. (5.1) and have

$$
\alpha=\frac{\tanh ^{-1} m_{1}}{m_{2}}=\frac{\tanh ^{-1} m_{2}}{m_{1}+m_{3}}=\frac{\tanh ^{-1} m_{3}}{m_{2}+m_{3}} .
$$

Notice that for $z>0$

$$
\frac{\mathrm{d}}{\mathrm{d} z}\left(\frac{\tanh ^{-1} z}{z}\right)>0 \text {. }
$$

Since $m_{2}>m_{1}$

$$
\frac{\tanh ^{-1} m_{1}}{m_{2}}=\frac{\tanh ^{-1} m_{2}}{m_{1}+m_{3}}>\frac{m_{2} \tanh ^{-1} m_{1}}{m_{1}\left(m_{1}+m_{3}\right)},
$$

F An elegant generalization of this theorem and a rigorous basis for eq. (4.1) have been established by Prof. C. Thompson ${ }^{7}$ ). 
therefore

$$
m_{1} / m_{2}>m_{2} /\left(m_{1}+m_{3}\right)
$$

which implies

$$
m_{2}-m_{1}<m_{1} m_{3} /\left(m_{1}+m_{2}\right)
$$

Similar analysis of the equations involving $\tanh ^{-1} m_{2}$ and $\tanh ^{-1} m_{3}$ gives

$$
\left(m_{3}-m_{2}\right) /\left(m_{2}-m_{1}\right)<m_{3} /\left(m_{1}+m_{3}\right)<1
$$

and the theorem is proved.

Theorem 5.2: For $\alpha>\alpha_{c}$ the (nontrivial) ferromagnetic solution of eq. (5.1) is stable.

Proof: From the free-energy functional $\psi$ given by eq. (4.2) the stability matrix analogous to $D$, defined by eq. (3.12), is

$$
E \equiv\left(\begin{array}{ccc}
\gamma_{1} & -1 & 0 \\
-1 & \gamma_{2} & -1 \\
0 & -1 & \gamma_{3}-1
\end{array}\right)
$$

where

$$
\gamma_{r}=1 / \alpha\left(1-m_{r}^{2}\right) \quad(r=1,2,3)
$$

Use of eq. (5.3) for $\alpha$ provides

$$
\gamma_{1}=m_{2} /\left(1-m_{1}^{2}\right) \tanh ^{-1} m_{1}
$$

and

$$
\gamma_{2}=\left(m_{1}+m_{3}\right) /\left(1-m_{2}^{2}\right) \tanh ^{-1} m_{2}
$$

One easily verifies the representation (for $0<u<1$ )

$$
\left(1-u^{2}\right)\left(\tanh ^{-1} u\right) / u=1-2 \sum_{0 \leqslant k \leqslant \infty} u^{2 k+2} /(2 k+1)(2 k+3),
$$

which implies the inequality

$$
\left(1-u^{2}\right)\left(\tanh ^{-1} u\right) / u<1 \quad(0<u<1),
$$


thus

$$
\begin{aligned}
& \gamma_{1}>m_{2} / m_{1}>1, \\
& \gamma_{2}>\left(m_{1}+m_{3}\right) / m_{2}>1,
\end{aligned}
$$

since $0<m_{1}<m_{2}<m_{3}$.

Now $E$ is positive-definite if and only if all principal minors have positive determinants. But $\gamma_{1}>1, \gamma_{2}>1$ establish that the determinants of the first two principal minors are positive. It only remains to be shown that $\operatorname{det} E>0$.

Let

$$
\begin{aligned}
& x=\alpha m_{2}>0, \\
& y=\alpha\left(m_{1}+m_{3}\right)>0, \\
& z=\alpha\left(m_{2}+m_{3}\right)>0,
\end{aligned}
$$

then with eq. (5.1)

$$
\begin{aligned}
& \tanh x=(x+y-z) / \alpha>0 \\
& \tanh y=x / \alpha>0 \\
& \tanh z=(z-x) / \alpha>0
\end{aligned}
$$

and

$$
\begin{aligned}
& \gamma_{1}=(\sinh 2 x) / 2(x+y-z), \\
& \gamma_{2}=(\sinh 2 y) / 2 x, \\
& \gamma_{3}=(\sinh 2 z) / 2(z-x) .
\end{aligned}
$$

Now

$$
\operatorname{det} E=\left(\gamma_{1} \gamma_{2}-1\right)\left(\gamma_{3}-1\right)-\gamma_{1} \text {, }
$$

which may be written

$$
\operatorname{det} E=E_{1} E_{2}-E_{3} \text {, }
$$


where

$$
\begin{aligned}
& E_{1}=[(\sinh 2 x)(\sinh 2 y)-.4 x(x+y-z)] / 4 x(z-x), \\
& E_{2}=[\sinh 2 z-2(z-x)] / 2(x+y-z), \\
& E_{3}=(\sinh 2 x) / 2(x+y-z) .
\end{aligned}
$$

For $w>0 \sinh w>w$; therefore, $E_{1}>1$ and the numerator of $E_{2}$ is $>0$. From eq. (5.18) the denominator of $E_{2}$ is $>0$; therefore, $E_{2}>0$ and

$$
\operatorname{det} E>[\sinh 2 z-\sinh 2 x-2(z-x)] / 2(x+y-z) .
$$

Since the denominator has just been shown to be positive, the stability will be proved if the numerator is also positive. That is, in fact, the case since

$$
(\sinh 2 z-\sinh 2 x) / 2(z-x)=1+\sum_{1 \leqslant k \leqslant \infty} a_{k},
$$

where

$$
a_{k}=\left[(2 z)^{2 k}+(2 z)^{2 k-1}(2 x)+\cdots+(2 x)^{2 k}\right] /(2 k+1) !>0 .
$$

Remark: For $M=2,4$ a similar analysis leads to the same conclusion, but for $M>6$ a rigorous proof seems more difficult.

We will now study eq. (5.1) numerically, and for that purpose the following transformation is useful:

$$
\begin{array}{ll}
n_{1}=m_{2}, & m_{1}=n_{1}+n_{2}-n_{3}, \\
n_{2}=m_{1}+m_{3}, & m_{2}=n_{1}, \\
n_{3}=m_{2}+m_{3}, & m_{3}=-n_{1}+n_{3} .
\end{array}
$$

In terms of the new variables and eq. (5.1)

$$
\begin{aligned}
& n_{1}=\tanh \alpha n_{2}, \\
& n_{2}=\tanh \alpha n_{1}+\tanh \alpha n_{3}, \\
& n_{3}=\tanh \alpha n_{2}+\tanh \alpha n_{3},
\end{aligned}
$$

or, equivalently,

$$
\begin{aligned}
& n_{1}=\tanh \alpha n_{2}, \\
& n_{2}=\tanh \alpha\left(n_{3}-\tanh \alpha n_{3}\right)+\tanh \alpha n_{3}, \\
& n_{3}=\tanh \alpha\left[\tanh \alpha\left(n_{3}-\tanh \alpha n_{3}\right)+\tanh \alpha n_{3}\right]+\tanh \alpha n_{3} .
\end{aligned}
$$


The latter system has the advantage that $n_{3}$ is given as a function of $n_{3}$ only for each $\alpha$, and graphical representation can be efficiently used to locate all solutions (stable as well as unstable). Iteration then enables one to pin down the stable solutions.

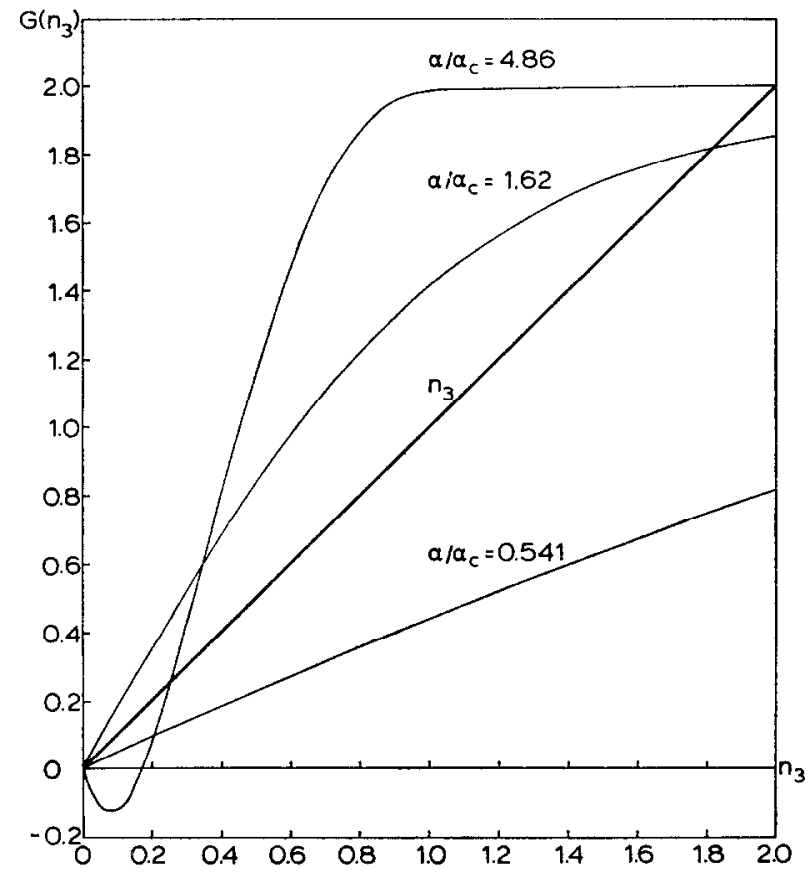

Fig. 1. The right-hand side of the last equation of the system (5.28) is denoted by $G\left(n_{3}\right)$ and is plotted for several values of $\alpha / \alpha_{c}$. Intersections of $G\left(n_{3}\right)$ with the line $n_{3}$ correspond to equilibrium solutions of eq. (5.28), but only the intersection farthest to the right for each $\alpha$ corresponds to the stable ferromagnetic equilibrium.

Fig. (1) shows the left- and right-hand sides of the last equation of the set (5.28) as functions of $n_{3}$ for three values of $\alpha / \alpha_{c}$. For large $\alpha / \alpha_{c}$ one sees two intersections for positive $n_{3}$. The smaller intersection merges with $n_{3}=0$ for $\alpha<1 / \lambda_{2}(1)$ $=1 /[2 \cos (3 \pi / 7)]$. However, that intersection leads to $n_{2}<0$ and does not correspond to the ferromagnetic solution. The ferromagnetic solution is precisely the larger $n_{3}$ intersection, and the corresponding magnetization curves as functions of $\alpha_{\mathbf{c}} / \alpha=\tau / \tau_{\mathbf{c}}$ are shown in fig. 2. The highest of the three curves is $m_{3}$, the next is $m_{2}$ and the lowest is $m_{1}$; all in agreement with the order established in theorem 4.2. The lower bound $m^{*}<m_{3}$ is not shown, but it is a very sharp bound which, as proved, lies above $m_{1}$.

Since, as mentioned earlier, eq. (2.6) is invariant under $|m\rangle \rightarrow-|m\rangle$, fig. 1 implies the existence of five solutions of eq. (5.1); viz., the up-down ferromagnetic 
pair which are stable, and another pair with $m_{1}=m_{6}>0, m_{2}=m_{5}>0$, $m_{3}=m_{4}<0$ in one solution and $m_{1}=m_{6}<0, m_{2}=m_{5}<0, m_{3}=m_{4}>0$ in the other. The latter solutions are unstable as is the trivial solution, and for $\alpha<1 / \lambda_{2}(1)$ [see eq. (4.6)] they merge with the trivial solution.

If one looks for solutions which violate the symmetry $m_{1}=m_{6}, m_{2}=m_{5}$, $m_{3}=m_{4}$, one finds at least one pair of solutions. In one solution $m_{1}=-m_{6}>0$, $m_{2}=-m_{5}>0, m_{3}=-m_{4}>0$ and $m_{1}=-m_{6}<0, m_{2}=-m_{5}<0, m_{3}$ $=-m_{4}<0$ in the other solution. They are both unstable and for $\alpha<1 / \lambda_{1}(-1)$ [see eq. (4.7)] they merge with the trivial solution. Thus, as the temperature is lowered from $\infty$, a pair of stable ferromagnetic solutions appear near the origin (bifurcation occurs), then at a lower temperature a pair of unstable, nonsymmetric, nonferromagnetic solutions appear near the origin. At a still lower temperature a pair of unstable, symmetric, nonferromagnetic solutions appear near the origin. The appearance of a pair occurs when $\alpha$ is the inverse of a positive eigenvalue of $\boldsymbol{A}$.

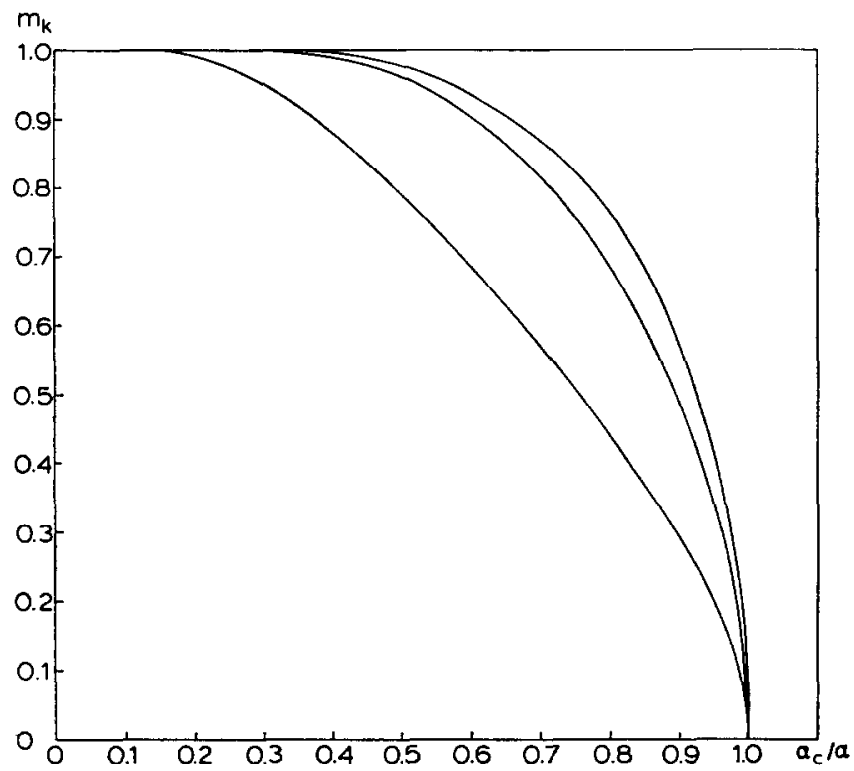

Fig. 2. The stable ferromagnetic solution of eq. (5.1) for $m_{1}\left(=m_{6}\right), m_{2}\left(=m_{5}\right), m_{3}\left(=m_{4}\right)$ versus $\alpha_{\mathrm{c}} / \alpha\left(=T / T_{\mathrm{v}}\right)$. The innermost chain has the largest magnetization $\left(m_{3}\right)$; whereas, the outermost chain has the smallest magnetization for each $\alpha$, all in agreement with eq. (4.15).

Since $A$ has $M / 2$ positive and $M / 2$ negative eigenvalues, there are three such occurrences for $M=6$; with the occurrence of the ferromagnetic pair when $\alpha$ is the inverse of the maximum eigenvalue of $A$. 
It should be emphasized that although we have proved the stability of the pair of ferromagnetic solutions for $2 \leq M \leq 6$ only, we conjecture that they are not only stable for arbitrary integer $M \geq 2$ but that they are the only stable real solutions to eq. (2.6) for $T<T_{\mathrm{c}}$. We also conjecture that the system of $M$ nonlinear equations, eq. (2.6), has at most $M+1$ real solutions (trivial solution included) for each $\alpha>0$. We have proved the latter conjecture for $M=2,3,4$ and have established the existence of seven solutions for $M=6$.

A cknowled gement. One of us (H.F.) thanks the Instituut voor Theoretische Fysica for generous hospitality and support during his sabbatical. We are grateful to Dr. C. Falk for kindly performing the computer analysis which led to figs. 1 and 2.

\section{REFERENCES}

1) See, e.g., Thompson, C. J., Mathematical Statistical Mechanics, Macmillan (New York, 1972) section $4-5$ and appendix $C$.

2) Griffiths, R.B., J. math. Phys. 8 (1967) 484.

3) Glauber, R. J., J. math. Phys. 4 (1963) 294.

4) Ruijgrok, Th.W. and Falk, H., Physica 78 (1974) 91.

5) See, e.g., Rutherford, D.E., Proc. Roy. Soc. Edinburgh, A LXII Part III (1947) 229.

6) Leff, H.S., J. math. Phys. 12 (1971) 569 and references therein.

7) Thompson, C. J., Physica, to be published. 